\title{
Direct observation of electrical charges at dislocations in GaAs by cross-sectional scanning tunneling microscopy
}

\author{
Ph. Ebert, ${ }^{\text {a) }}$ C. Domke, and K. Urban \\ Institut für Festkörperforschung, Forschungszentrum Jülich GmbH, 52425 Jülich, Germany
}

(Received 25 August 2000; accepted for publication 21 November 2000)

\begin{abstract}
We demonstrate the possibility of simultaneous determination of the type and electrical charge state of dislocations in GaAs by cross-sectional scanning tunneling microscopy (STM). The methodology is demonstrated for a dissociated perfect dislocation in highly Si-doped GaAs(110) surfaces. The STM images of the dislocation penetrating GaAs cleavage surface show that both partial dislocation cores as well as the stacking fault between the two partial dislocation cores are negatively charged.

(C) 2001 American Institute of Physics. [DOI: 10.1063/1.1341219]
\end{abstract}

Dislocations can affect, to a large degree, technological applications of semiconductors in electronic or optoelectronic devices ${ }^{1}$ by, e.g., introducing localized defect states in the band gap along the dislocation core. The existence of such defect states in dislocation cores has been predicted by theoretical calculations ${ }^{2-4}$ and experimental results were interpreted in terms of the presence of charges along dislocation lines. ${ }^{5-9}$ However, it has not been possible to identify the type of dislocation and determine simultaneously its electronic structure, such as the possible presence of localized defect states in the band gap. This correlated electronic and geometric knowledge is, however, crucial since there are indications that some dislocations may carry electrical charges, while others do not. ${ }^{10,11}$ Eventually, the knowledge of the type of dislocations, which carry charges, would provide the opportunity to improve device properties via an atomistic understanding of dislocations.

Here we demonstrate that cross-sectional scanning tunneling microscopy (STM) of dislocations penetrating through a GaAs(110) cleavage surface allows us to identify the type of dislocation and the presence of electrical charges at the dislocation core. STM has been used previously to study dislocations in $\mathrm{GaAs}^{12}$ and in InAs layers on $\operatorname{GaAs}(110),{ }^{13}$ however no traces of charges were found. Therefore, it has been concluded that dislocations in deformed Si-doped GaAs should be uncharged. ${ }^{12,14}$ In contrast, we observed a perfect dislocation dissociated into two partial dislocations which carry both negative charges in as-grown Si-doped GaAs. The stacking fault is also found to be charged.

For the experiments we used samples cut from a vertical Bridgeman grown GaAs crystal doped with (2.5-6) $\times 10^{19} \mathrm{~cm}^{-3} \mathrm{Si}$ as measured by secondary ion mass spectroscopy. The crystal also contained B as isoelectronic dopant in comparable concentrations. The carrier concentration was $(1-2) \times 10^{18} \mathrm{~cm}^{-3}$. In order to access grown-in dislocations by STM we cleaved the GaAs samples along a (110) plane in ultrahigh vacuum and imaged the resulting surface with atomic resolution. The samples were not subjected to any deformation to increase the dislocation concentration. Thus,

${ }^{a)}$ Electronic mail: p.ebert@fz-juelich.de we were able to observe some of the very rare dislocations present in as-grown GaAs crystals prepared for the use as standard substrates.

Figure 1(a) shows a constant-current STM image of the occupied states of a dislocation. The filled state maxima correspond to the occupied dangling bonds localized above the As atoms in the surface. ${ }^{15,16}$ Thus, the STM image can be interpreted as showing the positions of the As atoms in the surface, enabling us to determine the Burgers vector. The dislocation induces a "step" extending along the [1 10$]$ direction and ending at the points marked $\mathrm{P} 1$ and $\mathrm{P} 2$. The fact that the step is spatially limited shows that we are dealing in fact with two dislocation cores at the positions P1 and P2. The Burgers curcuit of the whole dislocation structure indicated in Fig. 1(a) yields that the two dislocation cores together have a Burgers vector $\mathbf{b}$ of $a / 2[1 \overline{10}]$. Figure 1(b) shows a magnified STM image of the lower (P2) dislocation core. The Burgers circuit shows that the dislocation P2 has an in plane component of the Burgers vector of $a / 4$ [1 $\overline{1} 0]$ $+a / 6[001]$. The vertical component of the Burgers vector can be deduced from the step height. The line profile in Fig. 1(c) taken at the row in [001] direction marked by the dashed line with the label $\mathrm{c}$ in Fig. 1(a) shows that the step is about $0.07 \mathrm{~nm}$ high. This height is $1 / 3$ of the height of a one atomic layer high step. Thus, the vertical component of the Burgers vector is $a / 12[\overline{1} \overline{1} 0]$ and the total Burgers vector of the dislocation P2 is $a / 6[1 \overline{2} 1]$. Dislocation P1 has a Burgers vector of $a / 6[2 \overline{1} \overline{1}]$.

The type of Burgers vectors of the two dislocations cores $\mathrm{P} 1$ and P2 indicate that both are partial dislocations arising from the dissociation of a perfect $a / 2$ [1 10$]$ dislocation. Thus, the stacking fault between the two partial dislocation cores gives rise to the step of $1 / 3$ step height and the lateral shift of the atom rows on both sides of the step. The dislocation lines of the partial dislocations should lie both in one of the two $\{111\}$ planes, whose intersection line with the (110) surface is the [1 $\overline{1} 0]$ direction (step direction). Figure 1 does not provide sufficient evidence to distinguish the two planes. However, as discussed below the asymmetric band bending suggests that the $\{111\}$ plane extending to the upper right side includes the dislocation lines.

At this stage we address the identification of localized charges. Localized charged on a (110) surface of III-V semi- 

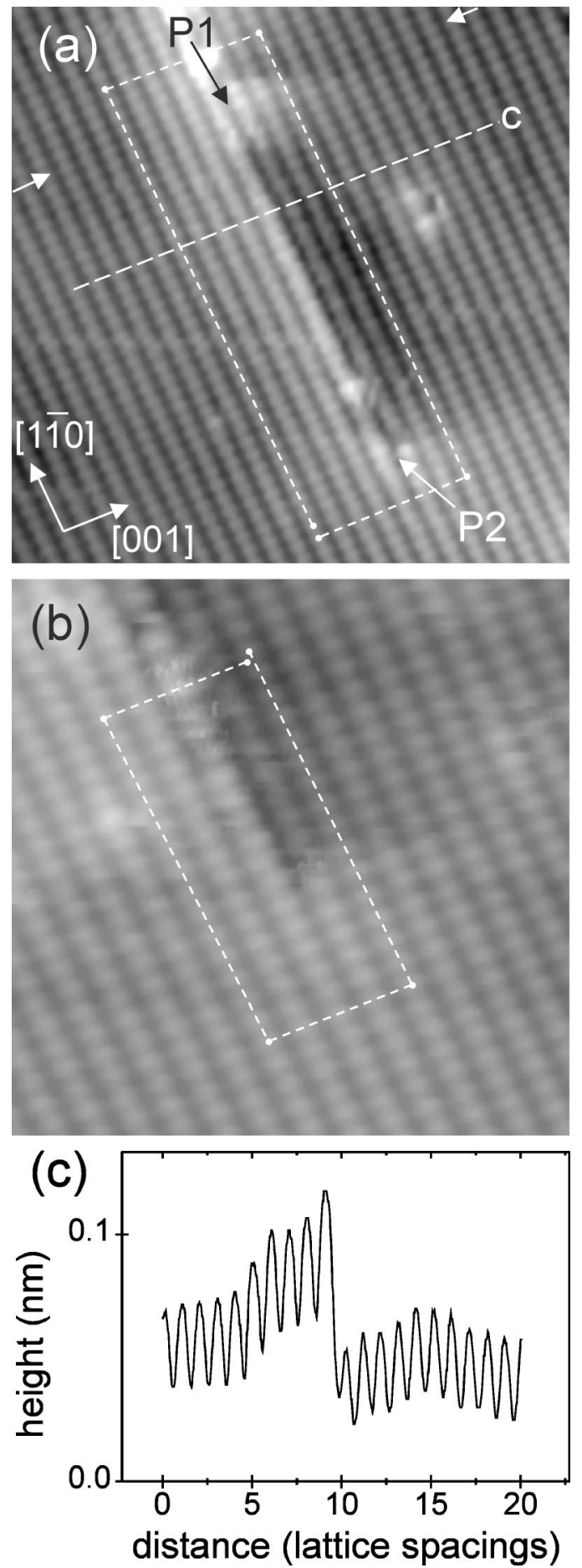

FIG. 1. Constant-current STM images of the occupied states of a dissociated perfect dislocation with a Burgers vector of $a / 2$ [1 10$]$. (a) Overview of the full dislocation dissociated into two partial dislocations (P1 and P2) with Burgers vectors of the type $a / 6\langle 211\rangle$. The image has been measured at $-2.0 \mathrm{~V}$ and $0.25 \mathrm{nA}$ current. (b) Magnified STM image of the lower partial dislocation core $(\mathrm{P} 2)$ obtained at $-2.2 \mathrm{~V}$ tunneling voltage. Indicated is the Burgers circuit. (c) Height profile along the dashed line $\mathrm{c}$ in frame (a). A step height of $1 / 3$ of that of a one atomic layer high step is visible.

conductors give rise to a localized band bending. ${ }^{17,18}$ This band bending can be imaged by STM. It induces a locally increased or decreased tunnel current, which is rather strongly voltage dependent. In constant-current STM images, the band bending leads to a height change. These chargeinduced contrast features were intensively investigated for charged point defects and dopant atoms in (110) cleavage Downloaded 15 Dec 2006 to 134.94.122.39. Redistribution subject

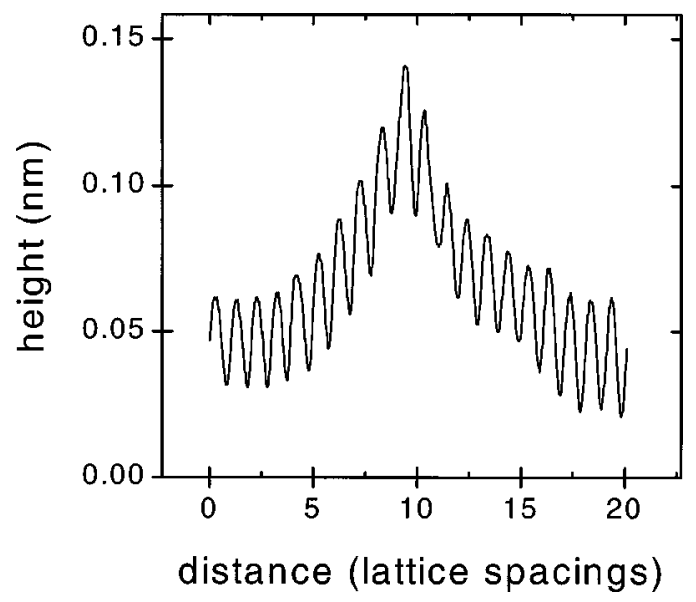

FIG. 2. Height profile through the dislocation core P1 along the atomic row in [001] direction marked by the two white arrows in Fig. 1(a). The height change indicates the presence of a band bending.

surfaces of most III-V semiconductors. ${ }^{19}$ Figure 2 shows a line profile through the partial dislocation core P1 along the [001] direction. It can be clearly recognized that at the dislocation core the height reaches a maximum. This elevation centered at the dislocation core suggests the presence of a band bending around the dislocation core. In order to exclude that the height change is only a geometric height change due to relaxation, we measured STM images at positive tunneling voltages, which show the empty states. Figure 3 shows that the contrast in empty state images is dark due to a wide depression at the two partial dislocation cores and at the stacking fault. Therefore, the height changes with the exception of the step are electronic effects and not geometric relaxations. Thus, the dislocation cores are indeed surrounded by a band bending. From the dark and bright contrast at positive and negative tunneling voltages we can con-

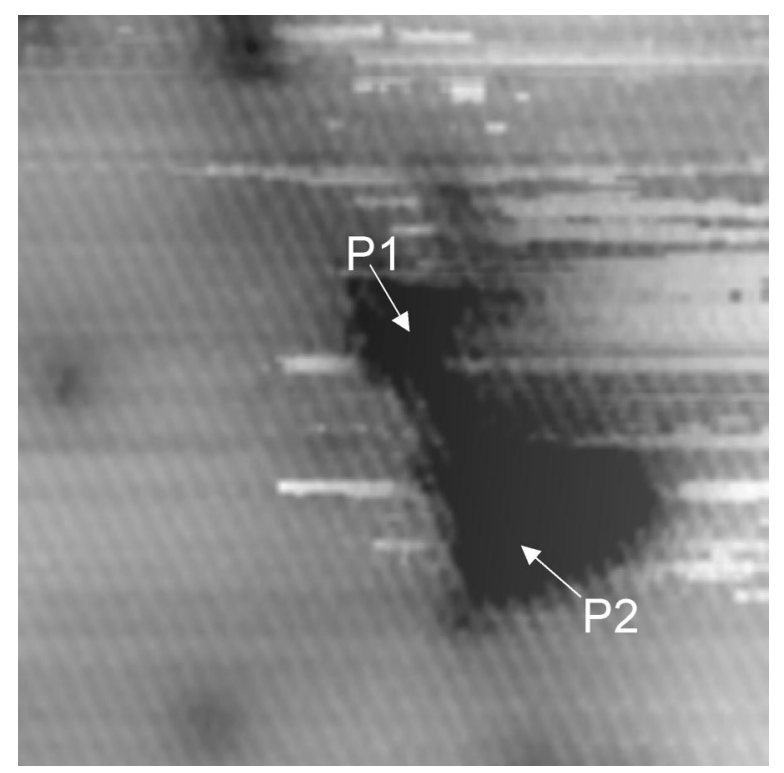

FIG. 3. Constant-current STM image of the empty states of the dislocation. The image has been acquired at $+1.8 \mathrm{~V}$ and $0.25 \mathrm{nA}$ and has the same orientation as those in Fig. 1. The wide dark depression indicates an upward band bending arising due to negative charges localized at the partial dislocation cores (approximate positions marked P1 and P2) and at the stacking fault. 
clude that the band bending is upward. ${ }^{20}$ This indicates that the two partial dislocations and the stacking fault are negatively charged.

The presence of a negative charge shows that the cores of the two partial dislocations must have localized defect states in the band gap. This suggests that the dislocation cores are not fully reconstructed to reach a charge neutrality.

Finally, the band bending is more extended in [001] than in [001] direction with respect to the stacking fault. If we consider that the dislocation lines must be localized in a $\{111\}$ plane, we can conclude that the partial dislocations are both lying in the $\{111\}$ plane whose intersection line with the cleavage surface is the $[1 \overline{1} 0]$ direction and which is entering into the bulk on the [001]-oriented side of the dislocation cores.

In conclusion, we demonstrated that cross-sectional scanning tunneling microscopy can be used to identify the presence of charges at dislocation cores with a simultaneous determination of the dislocation type, namely its Burgers vector. Similar measurements for a variety of different dislocation types may lead to a more detailed understanding of the electronic structures and reconstructions of dislocation cores in III-V semiconductors.

The authors thank K. H. Graf for technical support and L. Houben, M. Luysberg, U. Semmler, and C. Tillmann for helpful discussions.
${ }^{1}$ H. Alexander, in Dislocations in Solids, edited by F. R. N. Nabarro (North-Holland, Amsterdam, 1986), p. 113.

${ }^{2}$ F. Liu, M. Mostoller, V. Milman, M. F. Chisholm, and T. Kaplan, Phys. Rev. B 51, 17192 (1995).

${ }^{3}$ K. Leung, A. F. Wright, and E. B. Stechel, Appl. Phys. Lett. 74, 2495 (1999).

${ }^{4}$ J. R. Chelikowsky and J. C. H. Spence, Phys. Rev. B 30, 694 (1984).

${ }^{5}$ B. Chatterjee, S. A. Ringel, R. Sieg, R. Hoffman, and I. Weinberg, Appl. Phys. Lett. 65, 58 (1994).

${ }^{6}$ S. M. Lee, M. A. Belkhir, X. Y. Zhu, Y. H. Lee, Y. G. Hwang, and T. Frauenheim, Phys. Rev. B 61, 16033 (2000).

${ }^{7}$ D. Ferré and J. L. Farvacque, Rev. Phys. Appl. 25, 323 (1990).

${ }^{8}$ D. Gerthsen, Phys. Status Solidi A 97, 527 (1986).

${ }^{9}$ H. Nakata and T. Ninomiya, J. Phys. Soc. Jpn. 42, 552 (1977).

${ }^{10}$ R. Jaszek, J. J. Harris, and C. Roberts, J. Appl. Phys. 85, 959 (1999).

${ }^{11}$ R. Jones, A. Umerski, P. Sitch, M. I. Heggie, and S. Öberg, Phys. Status Solidi A 137, 389 (1993).

${ }^{12}$ G. Cox, D. Szynka, U. Poppe, K.-H. Graf, K. Urban, C. KisielowskiKemmerich, J. Krüger, and H. Alexander, Phys. Rev. Lett. 64, 2402 (1990).

${ }^{13}$ J. G. Belk, D. W. Pashley, C. F. McConville, J. L. Sudijono, B. A. Joyce, and T. S. Jones, Phys. Rev. B 56, 10289 (1997).

${ }^{14}$ G. Cox, D. Szynka, U. Poppe, K.-H. Graf, K. Urban, C. KisielowskiKemmerich, J. Krüger, and H. Alexander, J. Vac. Sci. Technol. B 9, 726 (1991).

${ }^{15}$ R. M. Feenstra, J. A. Stroscio, J. Tersoff, and A. P. Fein, Phys. Rev. Lett. 58, 1192 (1987).

${ }^{16} \mathrm{Ph}$. Ebert, B. Engels, P. Richard, K. Schroeder, S. Blügel, C. Domke, M. Heinrich, and K. Urban, Phys. Rev. Lett. 77, 2997 (1996).

${ }^{17}$ J. A. Stroscio, R. M. Feenstra, and A. P. Fein, Phys. Rev. Lett. 58, 1668 (1987).

${ }^{18}$ R. J. Hamers, J. Vac. Sci. Technol. B 6, 1462 (1988).

${ }^{19} \mathrm{Ph}$. Ebert, Surf. Sci. Rep. 33, 121 (1999).

${ }^{20}$ C. Domke, M. Heinrich, Ph. Ebert, and K. Urban, J. Vac. Sci. Technol. B 16, 2825 (1998). 